\title{
Students Conversation Management System
}

\author{
Abimbola R. Iyanda ${ }^{\text {a }}$, Olufemi D. Ninan ${ }^{\text {a }}$, Damilola J. Odejimi ${ }^{\text {a }}$ \\ ${ }^{a}$ Obafemi Awolowo University/Computer Science and Engineering Department, Ile-Ife, Nigeria
}

Received: 29 March 2018; Accepted: 13 June 2018; Published: 08 July 2018

\begin{abstract}
Customer service is an important area in the success of a system or a service. For services that have a relatively large customer base, the efficiency with which complaints are attended to becomes an issue. The Computer Centre of the Obafemi Awolowo University attends to students with various complaints majorly in relation to their e-portal accounts. Although efforts are in place to manage the crowd, there is still a major need for the complaint management service to save time and energy. The need for a system that can handle the enormous request and complaints of the undergraduate students of the institution is the thesis of this work. Design and implementation was done using the range of tools provided by the Microsoft Bot Framework. C\# Programming language was used to implement the decision algorithm. Online web services were used to handle natural language understanding and the Bot Connector to implement the Web Canvas. Microsoft Azure Service was used to host the web after which evaluations were drawn through surveys. Thus, this study projected an easier flow of operations involving logging of complaints by students.
\end{abstract}

Index Terms: Chatbot, Customer Service, Artificial Intelligence, Conversation, Natural Language Understanding.

(C) 2018 Published by MECS Publisher. Selection and/or peer review under responsibility of the Research Association of Modern Education and Computer Science.

\section{Introduction}

Chatbots have been around in various rudimentary forms for years, for example, Clippy, Microsoft's paper clip virtual assistant, Starbucks, Mastercard's Facebook Messenger etc. However, recently they have been taking off as advances in machine learning and artificial intelligence make them more versatile than ever [1]. It has since, been widely used or accepted and has seen an explosion of application from this concept in the past

* Corresponding author.

E-mail address: abiyanda@oauife.edu.ng, jninan@oauife.edu.ng,damilolaodejimi@gmail.com 
year. According to Lauren Kunze, there are two main categories of chatbots: utility chatbots and content-driven bots.

Utility gets something done following a prompt however content-driven chatbots focus on providing content. At a higher level, the more entertainment-related chatbots are able to answer a wide range of questions and get things done. It has been established that one can have small talk with Siri and Cortana, as well as getting things done, hence they are much harder to build [1].

\section{A. Customer Service and Chatbots}

In the past few years, chatbots have become useful partly due to the advent of many chat canvases and also due to the problems faced by customers on a website. It is described especially in some industries why people have begun to use chatbots. "Ref. [2]" reported that online customers experienced frustrations most commonly as a lack of basic information contained on everyday commercial websites, the inability to ask simple questions, poor quality of tools to provide a timely response.

Chatbot is used in education as an effective tool for online queries from students such as teaching assistant. It can deal with many routine queries but may not be able to handle all customer queries. In the recent time, the measured of customer service function is determined by how the number of customer calls and average handling time are reduced, which in turn reduced the cost of customer care.

The re-occurrence of the same problems with a large number of people having issues at the same time, and also the sluggishness to which the issues are logged or attended to due to necessary physical customer management (queues system, waiting time, physical recording or logging of issues and so on) is prominent locally with the Computer Centre. They attend to technical issues, especially relating to the e-portal of the University) in the Obafemi Awolowo University. There is a need for a model that allows students to log issues with ease and allow the relevant people to make sense of the data received (for instance, based on statistics).

The rest of the paper is structured as follows: Section 2 examines and presents a review of related works; Section 3 discusses the tools, data and the methodology employed in the development. Section 4 presents a discourse on the system evaluation and result while the paper concludes in Section 5.

\section{Related Works}

Customer service system includes a natural language device, a remote device remotely coupled to the natural language device over a network and a database. The database had a plurality of answers stored on it that are indexed to natural language keys [3].

The natural language device implements a natural language understanding system and receives a natural language question over the network from the remote device. The question is analyzed using the natural language understanding system. Based on the analysis, the database is then queried, an answer to the question is received based on the query, and the answer is provided to the remote device over the network [3].

Various tools exist to create personalized chatbot e.g. Rebot.me, ChatScript, Facebook's Bots for Messenger, Imperson, Pandorabots [4]. It can be inferred that the area of customer service will always need improvement in its response, efficiency and flexibility, hence the development of chatbot for customer services cannot be overemphasized.

The Implementation of chatbots in different application areas are available. For example, the Hotel Chatbot [5] have shown to bring great improvements in the domain. This chatbot simulates an intelligent discussion with hotels and guest houses customers who visit the webpage. It was confirmed from the data obtained by the authors, more than $56 \%$ of the conversations with the hotel chatbot (HC) function was informative, while $63 \%$ of users prefer to interact with the $\mathrm{HC}$ if it suggests at least one clickable option to choose as an alternative to typing.

Another example was the development of the smart system for Compaq customer service [6]. The work presents a smarts system that support a reduction in staffing level brought about as a result of the increase in the 
volume of support requests. This in turn gave a significance reduction in the cost of staff training and requirements. It was also an attempt to automate the process of customer service because of the increasingly competitive nature of the computer manufacturing industry.

"Ref. [7]" describes a system (Donna) which uses the information in the user's email account to stimulate setting and initiating meetings with his clients. The system was designed using pattern matching algorithm and implements web-based interactive Chabot which gets information about user's personal relationship with the client through machine learning tools.

"Ref. [8]" used an illustration of ALICE chatbot in terms of: Knowledge base and its pattern matching technique. This work Identified the main lack in ALICE and other chatbots as the manual developing of its knowledge to develop a chatbot system that reads from a corpus and converts it to ALICE knowledge base. It was established that a chatbot could be used as a tool to animate or visualize a corpus, a tool to learn/practice English, Arabic, Afrikaans or other languages [9], and a tool to access an information portal to provide answers to questions.

"Ref. [10]" constructs Freudbot using the open source architecture of AIML. AIML format is preferred due to the fact that it is closer to the markup structure used in annotated corpora and the simplicity in generating patterns/templates, and applying simple pattern matching technique [11].

"Ref. [12]" presents a novel approach for extracting high-quality <thread-title, reply> pairs as chat knowledge from online discussion forums so as to efficiently support the construction of a chatbot for a movie forum domain using a cascaded framework. 11,147 <thread-title, reply> pairs were extracted from 2,000 threads within two minutes. It is simply not feasible to have human experts encode a knowledge base of such size. Results from experiments conducted within a movie forum show the proposed approach is effective but that there is much room for future improvement of the quality.

"Ref. [13]" presents the intelligent QA (iQA) system for Arabic language which grows automatically as users ask questions thereby accumulation the question answer pairs in its database. The input question into the system was received in natural language and uses Google search engine, as information retrieval back-end. The questions retrieved from the document were then analyzed by a named-entity recognition tool in order to extract phrases of expected answer type.

"Ref. [14]" presents mobile based question answering system that acts as a personal assistant in learning and provides user with information on computers, software and hardware, book reviews using natural language. The knowledge base was created from the benchmark data set such as Amazon book reviews, 20newsgroup and Yahoo! Answer data set clustered with content specific clustering and displays the outcome in the form of snippets. Sentiment analysis was used to decrease the vocabulary gap among the user query and retrieved candidate answer solutions. The results of the proposed interface were evaluated using Precision, Recall, F1Score, Inverse precision and Inverse recall for the appropriate return of relevant answer. It was reported that the cuckoo search optimization with semantic similarity outperforms other methods.

"Ref. [15]" proposes a question answering system that uses the artificial immune algorithms, for finding the right answer in a knowledge database. In this paper, two scoring methods that use the genetic algorithm and artificial immune algorithm, are compared. Scoring is based on the question keywords matching, with the sentences in the knowledge base, and the question type matching with the knowledge base sentences type. After scoring the sentences in the knowledge base, the highest ranking sentence was displayed in the user interface. According to evaluations, the overall average accuracy of proposed system, have been significantly improved in comparison with genetic algorithm question answering (GA_QA). Search is based on the compatibility between question and answer types, as well as the overlap and non-overlap information between the question-answer pair. It was reported that results indicate significant increases in the accuracy of proposed system, in comparison with the previous systems

\section{Methodology}

Primary data were collected from Obafemi Awolowo University Computer Centre. Interviews were 
conducted with staff to acquire the necessary information suitable for the domain of this work. The category of the data consisted of student complaints (in order of how recurrent they are and the number of students that could face it at a time) and steps to resolving them.

The user experience and backend administration processes are shown in Fig. 1 which illustrates the basic surface interactions. The actors are the user and the administrator. The actions include "Open Chat Canvas", "Input text to initiate conversation", "Send Request", "Collate Data on Complaints" and "Acknowledge Solution (or Response) to Student".

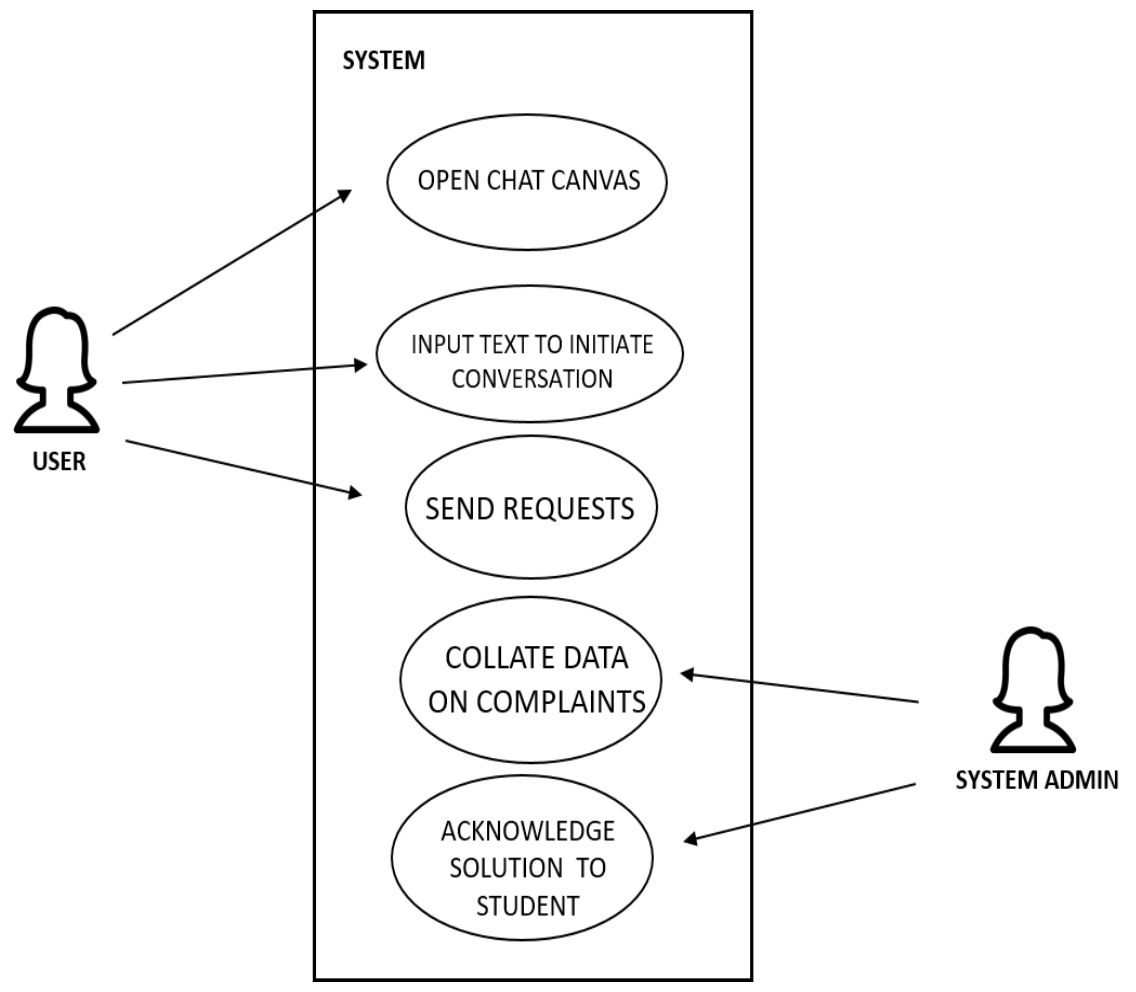

Fig.1. Use Case Diagram

\section{System Implementation}

The system was implemented using Microsoft Visual Studio Community 2015 (the Integrated Development Environment), the Bot-Builder Software Development Kit (SDK) and the Microsoft Bot Emulator. The chat control programming was done with the $\mathrm{C \#}$ programming language. The algorithm used was basically composed of 'if-else' conditional statements based on the collected data. The Microsoft's Language Understanding Intelligence Service (LUIS) web service was integrated at this point with the C\# code for understanding of the natural language.

The web which was used to understand the context of the complaints tendered over the chat canvas service was configured with sample statements, 'intents' and 'entities'. The context (intent) was mapped to an action or response (entity). The context mapping done with the LUIS was then integrated in the C\# code as part of the rules. The actions were then defined with the right responses and activities done by the system. The communication between each module of the system was achieved through Representative State Transfer 
(REST). REST was used because of its decoupled architecture, and lighter weight communications between producer and consumer; and also REST is a popular building style for cloud-based APIs.

Fig. 2 shows the screenshot of the implemented system and Figs. 3 and 4 show samples of simulated conversation between the developed system and a user when connected to bot via available channel.

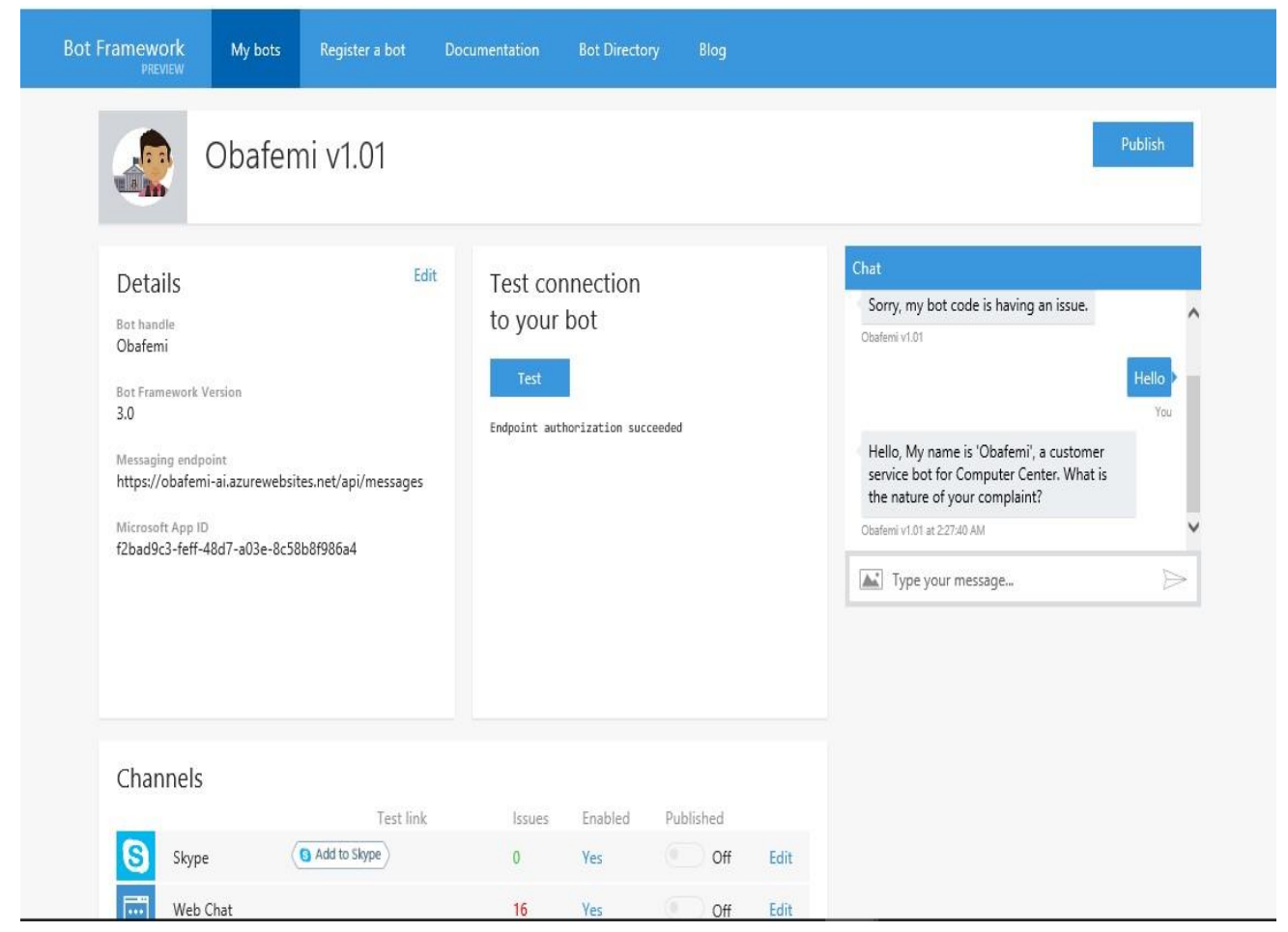

Fig.2. Screenshot of the Obafemi v1.01 Bot Profile on the Android Skype App

\section{Result and Discussion}

Testing was done after deployment on the Portal for the Bot Profile where a web chat canvas was provided for testing. The system was tested and correction made by the two (2) domain experts available. The results of the evaluation are shown in Table I as well as the average points. The 5-point scale ranges from 5 - 1 for each parameter in the survey. The parameters decided by the domain experts are Scalability, Naturalness of Response, Ease of Access, Functionality, Ease of Set Up/ Deploy-ability and Stability/ Reliability.

Two (2) domain experts tested and corrected the system after deployment on the Portal for the Bot Profile providing a web chat canvas. The parameters decided by the domain experts are shown in Table I as well as the average points (a scale of 0 - 5) given for each parameter in the survey. Scalability measures how the domain of implementation can be stretched or how more functionalities can be added across different domains;

Naturalness of Response measures how intelligent or human-like the responses were; Ease of Access measures how easy it was to access the system through the internet or the Chat canvases available.

Functionality measures how much the system served its purpose or how operational it was (i.e. how relevant the response was to the complaint); Ease of Set Up/Deploy-ability measures how easy it was to make releases/deploy the system and Stability/Reliability measures how reliable the system was during operation, based on the rate of failure. Due to the fact that the system is one that is scalable and can be improved from 
time to time with better dialogs, functionalities and features, the ease with which the system is updated and redeployed or published as an update became an evaluation factor, hence, the measure of its deploy-ability.

BOT: Hello, My name is 'Obafemi', a customer service bot for Computer Center. What is the nature of your complaint?

USER: Hello, I have issues login in to my e-portal account with my password.

BOT: Okay, so you want to change your password? Yes?

USER: Yes.

BOT: Okay, please input your username/matric number so we can log your issue

USER: mac/2013/100

BOT: We would need to verify your request and confirm it is really you. Okay?

USER: Okay

BOT: A mail has been sent to your registered email. Please verify and redirect through the password change procedure.

BOT: Alright, will there be anything else?

USER: when will e-portal be opened for PG Students?

BOT: I'm Sorry, didn't get that.

USER: Information on PG students registration

BOT: I'm sorry I do not understand your request, please rephrase or contact a human agent by mail: computercentre@gmail.com

BOT: Or visit room M15, Computer Centre, Faculty of

Technology Complex

BOT: Alright, will there be anything else?

USER: $N o$

BOT: Okay happy to be of service

Fig.3. Samples of Simulated Conversation I

BOT: Hello, My name is 'Obafemi', a customer service bot for
Computer Center. What is the nature of your complaint?
USER: i have registration issues
BOT: Okay, so if I get this right, you have issues registering
courses this session?
USER: yes
BOT: Okay, please input your username/matric number so we
can log your issue
USER: mee/2014/010
BOT: Okay, this is what you should do:
BOT: Write a letter to request for reopening of your course
registration page on the ePortal, as addressed below:
BOT: To: The Director, Computer Centre
$\quad$ Through: Chairman, Committee of Deans
$\quad$ Through: Dean of Faculty
Through: Head of Department
BOT: Write a letter to request for reopening of your course
registration page on the ePortal.
BOT: Take it to our Human agents at room M15, Computer
Centre, Faculty of Technology Complex for more confirmation.
BOT: Alright, will there be anything else?
USER: No
BOT: Okay happy to be of service

Fig.4. Samples of Simulated Conversation II 
Table 1. Result Analysis using the Domain Experts

\begin{tabular}{|c|c|c|}
\hline S/N & Parameter & Average Rating \\
\hline 1 & Scalability & 2.5 \\
\hline 2 & Naturalness of Response & 4 \\
\hline 3 & Ease of Access & 3 \\
\hline 4 & Functionality & 4 \\
\hline 5 & Ease of Set Up/ Deploy-ability & 4 \\
\hline 6 & Stability/Reliability & \\
\hline
\end{tabular}

The system evaluation was done using a total of Twenty-Five (25) people within the age of 18 - 26 years. The users available for the survey included fresh students (with generally no technical knowledge of this type of system) and non-freshmen students; especially the computer literate. The results of the evaluation are shown in Table II.

Table 2. Result Analysis from the Users

\begin{tabular}{|l|l|c|c|c|c|c|}
\hline & \multicolumn{1}{|c|}{ Parameters } & $\mathbf{5}$ & $\mathbf{4}$ & $\mathbf{3}$ & $\mathbf{2}$ & $\mathbf{1}$ \\
\hline 1 & Ease of Use & 6 & 11 & 5 & 2 & 1 \\
\hline 2 & Naturalness of Response & 2 & 4 & 12 & 5 & 2 \\
\hline 3 & Ease of Access & 5 & 15 & 3 & 2 & 0 \\
\hline 4 & Functionality & 4 & 9 & 7 & 3 & 2 \\
\hline 5 & Speed of Response & 6 & 13 & 5 & 1 & 0 \\
\hline 6 & $\begin{array}{l}\text { Comprehensiveness of } \\
\text { Response }\end{array}$ & 4 & 6 & 9 & 4 & 2 \\
\hline 7 & $\begin{array}{l}\text { Overall Customer } \\
\text { Experience }\end{array}$ & 0 & 9 & 10 & 5 & 1 \\
\hline
\end{tabular}

From the results of the survey, one of the areas of major improvement is the Naturalness of response. This was concluded due to the poor ratings given by the users in this criteria. About $25 \%$ i.e. six (6) out of twentyfive (25) respondents (who were majorly students that are not conversant with these kind of intelligent system) gave rating above three (3) points. Therefore, this brought about the conclusion that the area that lacked serious quality was the naturalness of response.

The aim of the evaluation is to compare the performance of the developed system with that of experts. The scales used ranges from 5 to 1. From Table II, it can be seen that the system is easy to access, easy to use and had a good response time. It is also shown that 17 out of $25(68 \%)$ respondents (for the ease of use criteria), 20 out of $25(80 \%)$ respondents (for the ease of access criteria) and 19 out of $25(76 \%)$ respondents (for the speed of response criteria) rated above 3 points. The high ratings given by the users (who were majorly students that are conversant with information technology and this kind of intelligent system) in those criteria brought about the conclusion.

\section{Conclusion}

This work developed for the education domain using the Computer Centre, OAU, Ile-Ife as a case study, is a web based application designed with different tools (mostly on the Microsoft Platform). The chatbot is accessible due to its integration with already established social networks (chat canvas or channels) by the Bot Connector. It employs the use of web services also to enhance intelligence and facilitate deployment. Due to the resources used, the system of making complaints with long queues and human effort was cut down to a very 
good minimum, as the majority of the process is automated.

In addition, more cognitive services can be integrated with the system to enhance its intelligence with the use of Microsoft Cognitive API's and other API's. Remote servers (e.g. available to the school or organization based on the domain) will help in monitoring and personal control of data, to create a closed network, instead of managing the Web Application with a Cloud Service (i.e. Microsoft Azure). The deployment of this system ensures a reduction in the cost of staff employment and training facilities.

\section{References}

[1] S. Carey. What is a chatbot, how do you build one, who is building them and are they any good? Tech World, <http://www.techworld.com/apps/what-is-chatbot-how-do-you-build-one-who-is-building-themare-they-any-good-3638740/>, Assessed on 7/12/2016.

[2] Myclever Agency. "MyClever Chatbots 2016 Whitepaper; a Consumer Research Study". <https://www.mycleveragency.com>, Assessed on 25/7/2016.

[3] R. G. Goldberg, R. R. Rosinski and T. Corp. Patent US5895466 - automated natural language understanding customer service system. 1997. <https://www.google.com/patents/US5895466>, Assessed on 12/12/2016.

[4] C. Jee. So you want to build a chatbot? Here are 6 of the best platforms for developers. Tech World, $<$ http://www.techworld.com/picture-gallery/apps/seven-platforms-for-developers-build-chatbots3639106/>, Assessed on 22/10/2016.

[5] M. Lasek and S. Jessa. "Chatbots for Customer Service on Hotels' Websites - Information systems in management" - Tom 2, Number 2 (2013). Biblioteka Nauki, Information Systems in Management, 2(2), pp 146-158. <http://yadda.icm.edu.pl/yadda/element/bwmeta1.element.desklight-c9f46380-f824-4f0597b5-5cf021d306be>, Assessed on 7/12/2016.

[6] T. L. Acorn and S. H. Walden. "SMART: Support management automated reasoning technology for Compaq customer service". In Proceedings of the fourth conference on Innovative applications of artificial intelligence, 1992, July (pp. 3-18). AAAI Press.

[7] N. Mhatre, K. Motani, M. Shah and S. Mali. "Donna Interactive Chat-bot acting as a Personal" Assistant. International Journal of Computer Applications, 140(10). 2016.

[8] B. AbuShawar and E. Atwell. Automatic Extraction of Chatbot Training Data from Natural Dialogue Corpora. RE-WOCHAT: Workshop on Collecting and Generating Resources for Chatbots and Conversational Agents - Development and Evaluation. Pp. 29-38. 2016.

[9] J. Jia "The study of the application of a web-based chatbot system on the teaching of foreign languages". In Society for Information Technology \& Teacher Education International Conference (pp. 1201-1207). Association for the Advancement of Computing in Education (AACE). 2004.

[10] B. Heller, M. Proctor, D. Mah, L Jewell, and B. Cheung. "Freudbot: An investigation of chatbot technology in distance education". In EdMedia: World Conference on Educational Media and Technology (pp. 3913-3918). Association for the Advancement of Computing in Education (AACE). 2005, June.

[11] B. A. Shawar and E. Atwell. "Using dialogue corpora to train a chatbot". In Proceedings of the Corpus Linguistics 2003 conference (pp. 681-690).

[12] J. Huang, M. Zhou and D. Yang. "Extracting Chatbot Knowledge from Online Discussion Forums". In IJCAI, 7:423-428. 2007, January.

[13] Waheeb Ahmed, Ajusha Dasan, Babu Anto P. "Developing an Intelligent Question Answering System", International Journal of Education and Management Engineering (IJEME), Vol.7, No.6, pp.50-61, 2017.DOI: 10.5815/ijeme.2017.06.06.

[14] K. Karpagam and A. Saradha. " A Mobile based Intelligent Question Answering System for Education Domain", International Journal of Information Engineering and Electronic Business (IJIEEB), Vol.10, 
No.1, pp. 16-23, 2018. DOI: 10.5815/ijieeb.2018.01.03.

[15] M. S. Fakhr, and M. S. Abadeh. "AISQA-An Artificial Immune Question Answering System”. International Journal of Modern Education and Computer Science, 4(3), 28. 2012.

\section{Authors' Profiles}
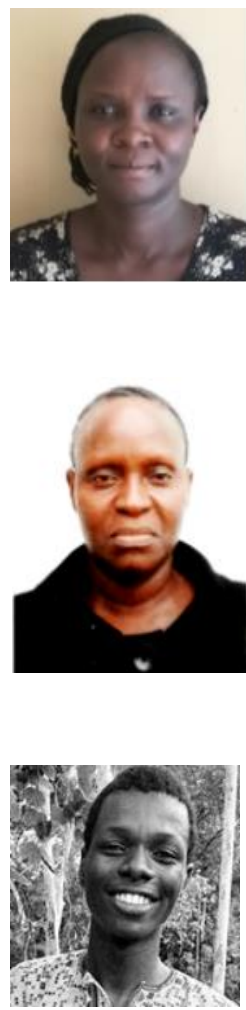

Dr. Abimbola R. Iyanda holds a B.Sc. degree in Computer Engineering, an M.Sc. and Ph. D. degrees in Computer Science from Obafemi Awolowo University, Ile-Ife, Nigeria. The thrust of her research is in the area of Computing and Intelligent Systems Engineering with focus on Speech and Language Engineering research aiming at domesticating computer technology and the computational rendering of indigenous ideas. She is a Member of the Nigerian Society of Engineers, Association of Professional Women Engineer in Nigeria, Council for the Regulation of Engineering in Nigeria and Nigeria Computer Society. Her present employment is with the Computer Science and Engineering Department, Obafemi Awolowo University, Ile-Ife, Nigeria.

Dr. Olufemi D. Ninan holds a B.Sc. degree in Applied Physics (Electronics) from the University of Lagos, M.Sc. and Ph. D. degrees in Computer Science from Obafemi Awolowo University, Ile-Ife, Nigeria. I am a Member of the Nigeria Computer Society, Association for Women in Science for the Developing World (OWSD). Her present employment is teaching and research as an academic staff with the Computer Science and Engineering Department, Faculty of Technology, Obafemi Awolowo University, Ile-Ife, Nigeria.

Mr. Damilola J. Odejimi holds a B.Sc. degree in Computer Science from Obafemi Awolowo University, Ile-Ife, Nigeria

How to cite this paper: Abimbola R. Iyanda, Olufemi D. Ninan, Damilola J. Odejimi,"Students Conversation Management System", International Journal of Education and Management Engineering(IJEME), Vol.8, No.4, pp.1-9, 2018.DOI: 10.5815/ijeme.2018.04.01 